\title{
The stress hyperglycemia ratio, a novel index of relative hyperglycemia, predicts short-term mortality in critically ill patients after esophagectomy
}

\author{
Zhili Xia ${ }^{1,2 \#, ~ T i n g ~ G u}{ }^{1 \#}$, Zhiyong Zhao ${ }^{1}$, Qian Xing ${ }^{1}$, Yaodong Zhang ${ }^{1}$, Zhongwei Zhang ${ }^{1}$, Biao Zhu ${ }^{1,2}$ \\ ${ }^{1}$ Department of Critical Care, Fudan University Shanghai Cancer Center, Shanghai, China; ${ }^{2}$ Department of Oncology, Shanghai Medical College, \\ Fudan University, Shanghai, China \\ Contributions: (I) Conception and design: Z Xia, T Gu; (II) Administrative support: B Zhu; (III) Provision of study materials or patients: Z Zhang; (IV) \\ Collection and assembly of data: Z Xia, T Gu; (V) Data analysis and interpretation: Z Xia; (VI) Manuscript writing: All authors; (VII) Final approval \\ of manuscript: All authors. \\ \#These authors contributed equally to this work. \\ Correspondence to: Biao Zhu. Department of Critical Care, Fudan University Shanghai Cancer Center, Shanghai, 200032, China; Department of \\ Oncology, Shanghai Medical College, Fudan University, Shanghai 200032, China. Email: zhubiaozs@126.com; Zhongwei Zhang. Department of \\ Critical Care, Fudan University Shanghai Cancer Center, Shanghai 200032, China. Email: spencezhang@hotmail.com.
}

Background: Postoperative mortality and severe complications are associated with both long-term blood glucose management and the severity of stress hyperglycemia. The purpose of this study was to assess the predictive value of a novel index, the stress hyperglycemia ratio (SHR), for short-term mortality in critically ill patients following esophagectomy.

Methods: A total of 356 patients who underwent esophagectomy for esophageal squamous cell carcinoma (ESCC) and were admitted to the intensive care unit (ICU) were included in this retrospective study. Based on the SHR values, patients were divided into low $(\mathrm{SHR}<1.14)$ or high $(\mathrm{SHR} \geq 1.14)$ groups in the overall and diabetic populations. The major outcomes of this study were the 30- and 90-day all-cause mortalities. We used Cox proportional hazard regression, Kaplan-Meier survival analysis, and competing risk regression models to analyze the relationships between risk factors and outcomes.

Results: The 30- and 90-day mortality in the high-SHR group were significantly higher compared to the low-SHR group in the total population (30-day: $1.3 \%$ vs. $10.5 \%, \mathrm{P}<0.001$; 90-day: $5.8 \%$ vs. $20.0 \%, \mathrm{P}<0.001$ ) and the diabetic population (30-day: $2.6 \%$ vs. $17.3 \%, \mathrm{P}=0.026$; 90 -day: $5.1 \%$ vs. $36.5 \%, \mathrm{P}<0.001$ ). After adjusting for covariables, the risk of the 30-day mortality [1.770 (1.442, 3.170)] and 90-day mortality [1.869 $(1.289,3.409)]$ remained significant $(\mathrm{P}=0.035, \mathrm{P}=0.045)$ in the total population. A similar result was observed in patients with diabetes [30-day: 1.642 (1.131, 2.710), $\mathrm{P}=0.015$; 90-day: 2.136 (1.254, 3.946), $\mathrm{P}=0.005$ ]. The Kaplan-Meier survival estimates for the 30-/90-day mortality also showed comparable results. The multivariable logistic regression analysis, including all glucose-related indices and the Acute Physiology and Chronic Health Evaluation (APACHE) II score, showed that SHR was independently correlated with the 30- and 90-day mortality; each 0.1-increase was related to a 3-4\% elevation in the 30-/90-day mortality [odds ratio (OR), 1.044; 95\% confidence interval (CI), 1.036-1.069; OR, 1.036; 95\% CI, 1.021-1.051].

Conclusions: In this study, we found that a relative increase in blood glucose, as quantified by the SHR $\geq 1.14$, was independently related to the higher 30-/90-day mortality in patients admitted to the ICU with severe complications following esophagectomy, while absolute hyperglycemia was not.

Keywords: Stress hyperglycemia; critical care; mortality; esophagectomy; cancer

Submitted Dec 14, 2021. Accepted for publication Feb 16, 2022.

doi: 10.21037/jgo-22-11

View this article at: https://dx.doi.org/10.21037/jgo-22-11 


\section{Introduction}

Esophageal cancer (EC) is the eighth most common type of cancer worldwide, with more than 480,000 newly diagnosed patients every year. Despite successful perioperative management, the administration of improved surgical techniques, undergoing primary surgery, and having positive microscopic resection edges (R1), EC patients have a much lower survival rate than expected, with a 5-year survival rate of less than $40 \%(1-5)$. Therefore, it is essential to explore valuable factors to predict the prognosis of EC patients and provide early treatments.

In diabetic patients, poor glucose management has adverse clinical outcomes, including postoperative site infections, poor wound healing, and prolonged hospitalization (6-12). Meanwhile, acute hyperglycemia can occur in people suffering from a severe disease as a result of physiological stress, even in the absence of previous diabetes. Stress-induced hyperglycemia has also been associated with a significantly greater risk observed in patients without a history of diabetes compared to those with a known diagnosis of diabetes (8,13-16). Previously, the majority of research has used admission blood glucose (ABG) levels or random glucose concentrations to determine the degree of stress hyperglycemia $(10,11,17,18)$. In 2004, Vriesendorp's team reported that postoperative hyperglycaemia was associated with increased length of inhospital stay in patients undergoing highly invasive surgery for EC (19). However, the stress-related blood glucose elevation may be caused by poor chronic blood glucose control, physiological reaction of acute illness or both, and it is incomplete to evaluate the prognosis only by absolute hyperglycemia, which ignores the background blood glucose. Roberts et al. (20) recently introduced the stress hyperglycemia ratio (SHR), a novel index that is calculated by dividing the absolute blood glucose by the estimated average glucose obtained from glycosylated hemoglobin $\left(\mathrm{HbA}_{1 \mathrm{c}}\right)$ : [glucose $\left.(\mathrm{mg} / \mathrm{dL}) / 18\right] /\left[\left(1.59^{*} \mathrm{HbA}_{1 \mathrm{c}}\right)-2.59\right]$. Additionally, Roberts' team found that the SHR was a more precise critical disease (in-hospital mortality or critical care) predictive marker than absolute glucose because it controls background glucose levels.

A relative increase in blood glucose refers to a sharp increase in blood glucose concentration compared with the background levels due to the inflammatory and neurohormonal derangements of the stress response. Surgical trauma induces stress hormone secretion, which is mainly mediated by glucagon, in turn promotes insulin resistance and oxidative stress, and finally leads to the blood glucose shoot up. According to recent studies, relative hyperglycemia is more correlated with adverse outcomes than an increase in absolute blood glucose concentration (20-23). However, on the basis of clinical observation, patients with critical postoperative complications after esophagectomy have varying degrees of postoperative hyperglycemia, regardless of whether they had diabetes or not. The intractable inpatient hyperglycemia will lead to a protracted disease course, and greatly increase the length of hospitalization and medical expenses. Designing personalized blood glucose management strategies that are based on the different background glucose concentrations, may, at least in part, benefit these patients. However, previous studies have rarely focused on the relationship between an increase in blood glucose and the prognosis of postoperative critically ill patients with EC based on background blood glucose concentration. The purpose of this study was to determine whether SHR could be used to predict adverse outcomes of severe complications in individuals with variable baseline blood glucose levels following esophagectomy.

We present the following article in accordance with the STARD reporting checklist (available at https://jgo. amegroups.com/article/view/10.21037/jgo-22-11/rc).

\section{Methods}

\section{Study population}

This retrospective study was carried out at the Fudan University Shanghai Cancer Center from January 2018 to December 2020. The study analyzed data from 428 consecutive patients who underwent potential curative esophagectomy for esophageal squamous cell carcinoma (ESCC) and were admitted to the intensive care unit (ICU) due to acute postoperative complications. Preoperative HbA1c levels were determined in all patients. The ICU team documented the reasons for admission to the ICU. The following criteria were used to exclude patients: hypoglycemia, diabetes ketoacidosis, or hyperosmolar hyperglycemia syndrome as the glycemic-related primary reasons for ICU admission $(n=4)$, incomplete laboratory data on preoperative HbA1c $(n=31)$, glucose levels within 24 hours of operation ( $n=2)$, and no Acute Physiology and Chronic Health Evaluation II (APACHE II) score $(n=1)$ assessment. Additionally, patients who had conditions that affect HbA1c levels were excluded from the study, including 
anemia [defined as hemoglobin less than $10 \mathrm{~g} / \mathrm{dL}(\mathrm{n}=17)$ ], serum creatinine levels $>2.0 \mathrm{mg} / \mathrm{dL}$ [indicating overt renal failure $(n=11)$ ], and patients receiving hemodialysis or peritoneal dialysis $(\mathrm{n}=6)$. Finally, 356 patients were included.

The study was conducted in accordance with the Declaration of Helsinki (as revised in 2013). This study was approved by the Ethics Committees of the Fudan University Shanghai Cancer Center, Shanghai, China. Because this study used retrospective data routinely collected during health screening process, the ethics committee exempted the requirement of obtaining informed consent.

\section{Data collection}

The baseline information and perioperative variables of all included patients were obtained. The baseline data included the following: age, body mass index (BMI), American Society of Anesthesiologists (ASA) physical status score, and preoperative laboratory data. The Charlson Comorbidity Index (24) was calculated on the basis of diabetes mellitus $(\mathrm{DM})$, cerebrovascular disease, congestive heart failure, myocardial infarction, chronic pulmonary disease, peripheral vascular disease, rheumatic disease, dementia, leucocythemia, lymphoma, metastatic solid tumour, mild and severe liver disease, renal disease, peptic ulcer disease, paraplegia, and acquired immune deficiency syndrome (AIDS). Preoperative laboratory findings included HbA1c, ABG levels, serum creatinine $(\mathrm{Cr})$ and hemoglobin $(\mathrm{Hb})$, the blood glucose-related indexes determined within 24 hours after operation, APACHE II score, and the need for vasopressors or mechanical ventilation in the ICU. Each patient's APACHE II score was calculated to determine the disease-specific risk of death [as defined by Knaus et al. (25)].

The postoperative complications included the following: pulmonary complications [defined as the presence of one or more of the following: acute respiratory distress syndrome (ARDS), pneumothorax and respiratory failure requiring more than 48 hours of ventilatory support], pneumonia cardiovascular complications (including severe arrhythmia, heart failure, myocardial infarction, cardiogenic pulmonary edema and pulmonary embolism), anastomotic leakage (defined as any clinical signs of leakage and confirmation of fistula by imaging or endoscopy, with or without mediastinitis, pleural empyema/abscess, tracheobronchial lesion, and anastomotic bleeding), recurrent laryngeal nerve palsy (confirmed by bedside visualization of the glottis), chylothorax (diagnosed using the chylous test for thoracic fluid drainage), and sepsis (determined by visually identified infection or positive microbial culture, and clinical signs of systemic inflammatory response syndrome (SIRS) occurring during hospitalization within 30 days of surgery), were recorded.

In addition, the length of hospital and ICU stay was also recorded. The main outcome in this study was the 30 - and 90-day all-cause mortality. All variables were acquired retrospectively by reviewing the electronic medical records. Postoperatively, all patients were followed-up for 3 months via telephone or outpatient follow-up system. After 3 months of follow-up, the patients were considered survivors $(26,27)$.

\section{Calculation of relative hyperglycemia}

The Nathan equation was utilized to determine the average blood glucose concentration during the prior 3 months using $\mathrm{HbAlc}$; the estimated average glucose concentration $(\mathrm{mmol} / \mathrm{L})$ was calculated as $\left(1.59^{*} \mathrm{HbA1c}\right)-2.59(28)$. The SHR was used to define relative hyperglycemia; it was determined by dividing the first obtained plasma glucose concentration detected within 24 hours after surgery by the estimated average blood glucose (20). In the Roberts's original study, 1.14 and 1.38 corresponding to the average SHR values of fourth and fifth SHR quintile with higher mortalities and an SHR $>1.14$ is considered a more reliable predictor of adverse outcomes than absolute blood glucose levels in the presence or absence of previously known diabetes. The threshold of 1.14 was used in a study of patients undergoing orthopedic surgery, and SHR greater than 1.14 was confirmed to be associated with poor prognosis (29). In this study, patients who were being treated for a prior positive diabetic history or a preoperative HbA1c level $\geq 6.5 \%$ were considered to have DM.

\section{Study cohort}

On the basis of the SHR values, we categorized all patients into the low (SHR $<1.14$ ) or high (SHR $\geq 1.14$ ) SHR groups. The diabetic population were divided into subgroups based on a SHR value $<1.14$ and $\geq 1.14$.

\section{Statistical analysis}

The median (interquartile range) was used to express continuous variables. Comparisons were performed using the Kruskal-Wallis test. The chi-square test was applied to assess categorical variables that were provided as counts 
Table 1 Basic characteristics and postoperative outcomes in the total and diabetic populations based on the stress hyperglycemia ratios

\begin{tabular}{|c|c|c|c|c|c|c|}
\hline Variable & \multicolumn{3}{|c|}{ Total population } & \multicolumn{3}{|c|}{ Diabetic population } \\
\hline Age (years) & 65 [56-72] & $67[57-74]$ & 0.786 & $64[57-72]$ & 67 [63-73] & 0.018 \\
\hline BMI $\left(\mathrm{kg} / \mathrm{m}^{2}\right)$ & $23.18(20.90,25.21)$ & $23.36(20.64,25.39)$ & 0.986 & $22.8(20.9,24.5)$ & $23.38(21.36,25.45)$ & 0.073 \\
\hline ASA classification, $n(\%)$ & & & 0.842 & & & 0.064 \\
\hline II & $124(80.0)$ & $164(78.1)$ & & $30(76.9)$ & $35(67.3)$ & \\
\hline III & $18(11.6)$ & $27(12.9)$ & & $6(15.4)$ & $13(25.0)$ & \\
\hline APACHE II & 8 [6-12] & $10[7-14]$ & 0.006 & $7[5-9]$ & 8 [6-12] & 0.013 \\
\hline PBG (mmol/L) & $6.84(6.21-7.47)$ & $12.72(9.43-15.82)$ & 0.022 & $11.62(7.91-16.43)$ & $14.49(10.59-21.13)$ & 0.483 \\
\hline Vasopressor, n (\%) & $23(14.8)$ & $34(16.2)$ & 0.629 & $8(20.5)$ & $15(28.8)$ & 0.114 \\
\hline Ventilator, n (\%) & $21(13.5)$ & 55 (26.2) & $<0.001$ & $10(25.6)$ & $17(32.7)$ & 0.048 \\
\hline Laryngeal nerve palsy (\%) & $24(15.5)$ & $27(12.8)$ & 0.782 & $4(10.2)$ & $8(15.4)$ & 0.391 \\
\hline Chylothorax (\%) & $3(1.9)$ & $6(2.9)$ & 0.471 & $1(2.6)$ & $2(3.8)$ & 0.424 \\
\hline Sepsis (\%) & $7(4.5)$ & $15(7.1)$ & 0.611 & $3(7.7)$ & $5(9.6)$ & 0.384 \\
\hline Pulmonary complications (\%) & $60(38.7)$ & $81(38.6)$ & 0.761 & $16(41.0)$ & $14(26.9)$ & 0.057 \\
\hline Anastomotic leakage (\%) & $36(23.2)$ & $69(32.9)$ & 0.170 & 7 (17.9) & $25(48.1)$ & $<0.001$ \\
\hline Cardiovascular diseases (\%) & $7(4.5)$ & $15(7.1)$ & 0.482 & $2(5.1)$ & $7(13.5)$ & 0.046 \\
\hline
\end{tabular}

Patients were grouped based on SHR values ( $<1.14$ or $\geq 1.14)$. ASA, American Society of Anesthesiologists; BMI, body mass index; APACHE II, Acute Physiology and Chronic Health Evaluation; PBG, Postoperative blood glucose; SHR, Stress hyperglycemia ratio.

(frequencies). Kaplan-Meier (KM) survival analysis and the Cox proportional hazard regression model were utilized to assess the relationship between the groups and the 30- and 90-day mortality rates. Univariate and multivariate analyses were carried out using Cox regression analysis. Variables with a $\mathrm{P}$ value $<0.05$ in the univariate analysis were included in the multivariable Cox regression analysis. The odds ratio (OR) and its 95\% confidence interval (CI) were estimated. The area under the receiver operating characteristic (AUROC) curve was used to assess the variables' potential to predict mortality. IBM SPSS Statistics for Windows, Version 20.0, was used to perform all statistical analyses (IBM Corp., Armonk, NY, USA). A two-tailed P value
$<0.05$ was considered statistically significant.

\section{Results}

The 356 patients were allocated into groups as follows: in the general population, low $(\mathrm{n}=155)$ and high $(\mathrm{n}=210)$ SHR groups, and in the diabetes, low $(n=39)$ and high $(\mathrm{n}=52)$ SHR groups. Table 1 summarizes the patients' baseline characteristics. APACHE II score $(\mathrm{P}=0.006$, $\mathrm{P}=0.013)$ and ventilator usage $(\mathrm{P}<0.001, \mathrm{P}=0.048)$ were significantly different between the groups in both the general and diabetic populations. Also, there were no significant differences in age, BMI, ASA classification, 
Table 2 HRs (95\% CIs) for in-hospital complications between the general and diabetic populations

\begin{tabular}{|c|c|c|c|c|c|c|}
\hline In-hospital complications & SHR $<1.14$ & $\mathrm{SHR} \geq 1.14$ & Unadjusted HR $(95 \% \mathrm{Cl})$ & $P$ value & Adjusted HR (95\% Cl) & $P$ value \\
\hline 30-day mortality & $2(1.3)$ & $22(10.5)$ & $1.893(1.615-3.650)$ & 0.004 & $1.770(1.422-3.170)$ & 0.035 \\
\hline 90-day mortality & $9(5.8)$ & $42(20.0)$ & $1.934(1.563-3.689)$ & $<0.001$ & 1.869 (1.289-3.409) & 0.045 \\
\hline \multicolumn{7}{|l|}{ Diabetic population } \\
\hline 90-day mortality & $2(5.1)$ & $19(36.5)$ & $1.745(1.211-3.383)$ & 0.002 & $2.136(1.254-3.946)$ & 0.005 \\
\hline Anastomotic leakage (\%) & 7 (17.9) & $25(48.1)$ & $1.563(1.103-3.269)$ & 0.004 & $1.982(1.246-3.541)$ & 0.002 \\
\hline Cardiovascular diseases (\%) & $2(5.1)$ & $7(13.5)$ & $1.683(1.372-3.024)$ & 0.003 & $1.492(1.879-2.142)$ & 0.038 \\
\hline
\end{tabular}

To calculate hazard ratios (HRs) with 95\% confidence intervals (Cls), we used a Cox proportional hazards regression model (Cls). SHR, stress hyperglycemia ratio.

Charlson Comorbidity Index, HbA1c, vasopressor use, postoperative complications (chylothorax, sepsis, pulmonary complication), or median length of hospital stay between the high and low SHR groups in both the general and diabetic populations. In the total population, there was a marked difference in PBG between the low and high SHR groups (6.84 vs. $12.72 \mathrm{mmol} / \mathrm{L}, \mathrm{P}=0.022)$; however, there was no significant variation among the diabetic population (11.62 vs. $14.49 \mathrm{mmol} / \mathrm{L}, \mathrm{P}=0.483$ ). In addition, there were significant differences in anastomotic leakage $(17.9 \%$ vs. $48.1 \%, \mathrm{P}<0.001)$ and cardiovascular diseases $(5.1 \%$ vs. $13.5 \%, \mathrm{P}=0.046)$ between the low and high SHR groups in the diabetic population; however, there was no significant difference among the total population $(23.2 \%$ vs. $32.9 \%$, $\mathrm{P}=0.170 ; 4.5 \%$ vs. $7.1 \%, \mathrm{P}=0.482$ ).

The median length of ICU stay in the total population was 3 (range, 1-6) and 5 (range, 1-11) days in the high and low SHR groups, respectively $(\mathrm{P}=0.001)$. In contrast, there was no notable difference in the median length of ICU stay between the low and high SHR groups in the diabetic population $\{4[2,11]$ vs. $5[2,14], \mathrm{P}=0.231\}$. The 30-day mortality rate in the high SHR group was significantly higher than that in the low SHR group, both in the total population $(1.3 \%$ vs. $10.5 \%, \mathrm{P}<0.001)$ and the diabetic population $(2.6 \%$ vs. $17.3 \%, \mathrm{P}=0.026)$. Also, the 90 -day mortality rate in the high SHR group was significantly higher than that in the low SHR group, both in the general population $(5.8 \%$ vs. $20.0 \%, \mathrm{P}<0.001)$ and the diabetic population $(5.1 \%$ vs. $36.5 \%, \mathrm{P}<0.001$ ) (as shown in Table 1 ).

As illustrated in Table 2, compared with the low SHR group, the high SHR group had a hazard ratio (HR) $(95 \%$ CI) of $1.893(1.615,3.650)$ and $1.934(1.563,3.689)$ for the 30-day mortality and 90-day mortality, respectively, in the total population. After adjusting for APACHE II score, PBG, ventilator use, anastomotic leakage, cardiovascular diseases and length of ICU stay, the risk of the 30-day mortality $[1.770(1.442,3.170)]$ and 90 -day mortality [1.869 (1.289, 3.409)] was still significant $(\mathrm{P}=0.035, \mathrm{P}=0.045)$. Similarly, in the diabetic population cohort, the 30-day mortality risk was greater in the high SHR group (64\%) than in the low SHR group $(\mathrm{P}=0.015)$ after adjusting for covariables (APACHE II score, PBG, and ventilator use). The 90-day mortality was nearly double in the diabetic population with a high SHR than the low SHR group [2.136 $(1.254,3.946)]$ after adjusting for covariables $(\mathrm{P}=0.005)$. Moreover, the rates of anastomotic leakage [1.982 (1.246, 3.541)] and cardiovascular diseases [1.492 (1.879, 2.142)] were significantly higher in high SHR group. The KaplanMeier estimates of the 30-/90-day mortality also showed comparable results (Figure 1).

In univariate analyses, $\mathrm{ABG}(\mathrm{OR}=1.083, \mathrm{P}=0.022), \mathrm{PBG}$ $(\mathrm{OR}=1.036 ; \mathrm{P}<0.001)$, SHR $(\mathrm{OR}=1.096 ; \mathrm{P}=0.001)$, and APACHE II score $(\mathrm{OR}=1.437 ; \mathrm{P}<0.001)$ were significant predictors of 30-day mortality (Table 3). After adjustment for APACHE II score and the above glucose-related indices, SHR was found to be significantly associated with 30-day mortality $(\mathrm{OR}=1.044 ; \mathrm{P}<0.001)$, whereas $\mathrm{PBG}$ was not ( $\mathrm{OR}=1.012 ; \mathrm{P}=0.130)$. The same result is shown in Table 4; after adjustment for APACHE II score and glucose-related indices, SHR was markedly associated with 90-day mortality (OR =1.036; $\mathrm{P}=0.013)$, whereas $\mathrm{PBG}$ was not $(\mathrm{OR}=1.009$; $\mathrm{P}=0.210)$.

The 90-day mortality AUROC curve of SHR was 0.733 (0.654-0.812). As illustrated in Figure 2, the AUROC curve 

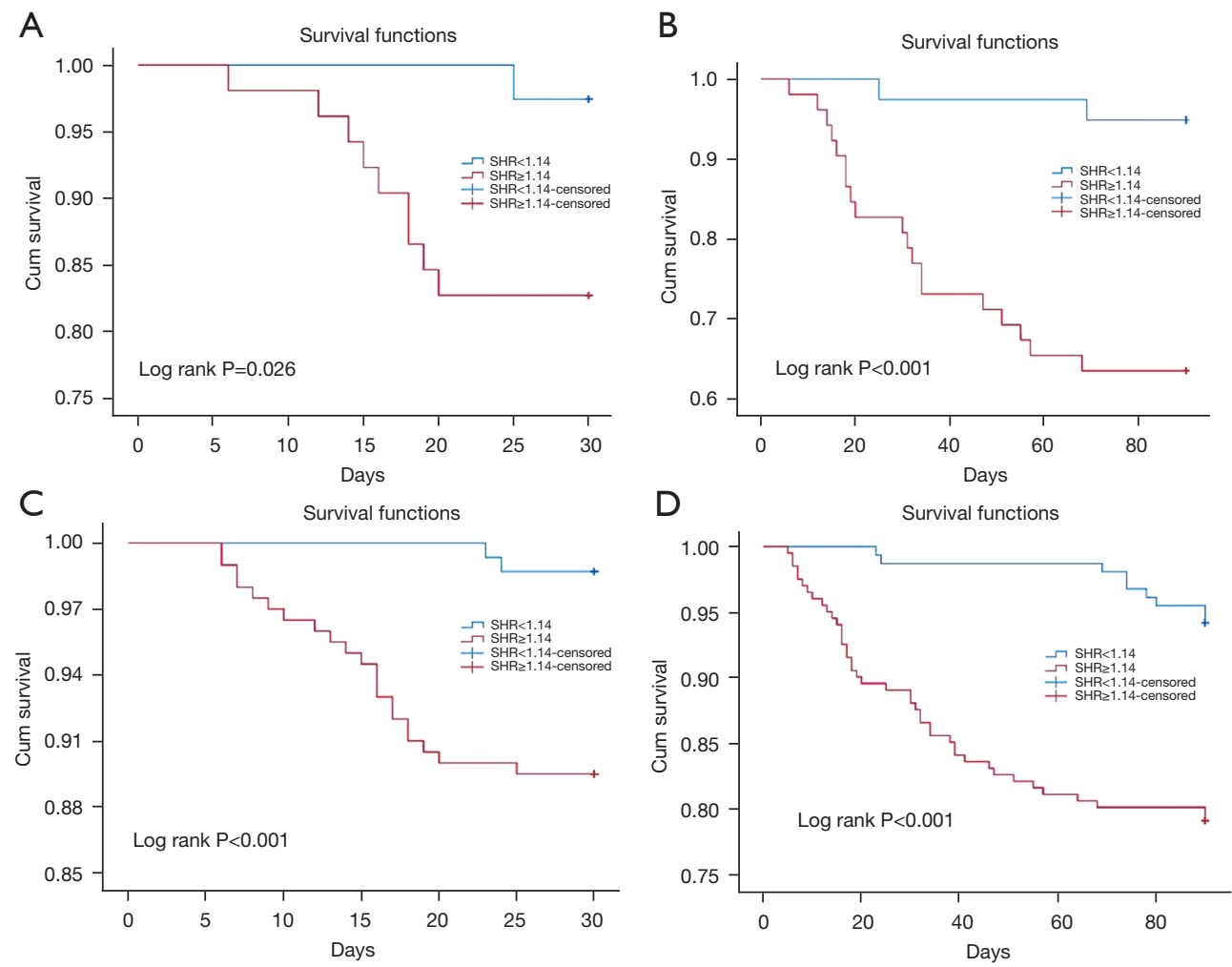

Figure 1 Kaplan-Meier survival curves demonstrating the probability of survival in the various groups. A P value for the Log-rank test $<0.05$ showed that patients in the high SHR group had a lower survival probability. (A) 30-day survival probability in the diabetic population; (B) 90-day survival probability in the diabetic population; (C) probability of survival for the total population after 30 days; (D) probability of surviving for 90 days in the overall population. SHR, stress hyperglycemia ratio.

Table 3 Univariable and multivariable regression model results showing the relationship between the selected variables and 30-day mortality

\begin{tabular}{|c|c|c|c|c|}
\hline Risk factor & \multicolumn{2}{|c|}{ Univariable analysis } & \multicolumn{2}{|c|}{ Multivariable analysis } \\
\hline $\mathrm{ABG}, \mathrm{mmol} / \mathrm{L}$ & $1.083(1.036-1.293)$ & 0.022 & $0.927(0.758-1.281)$ & 0.864 \\
\hline $\mathrm{PBG}, \mathrm{mmol} / \mathrm{L}$ & $1.036(1.012-1.243)$ & $<0.001$ & $1.012(0.807-1.039)$ & 0.130 \\
\hline $\mathrm{HbA} 1 \mathrm{c}$, per $1 \%$ & $1.772(1.224-2.565)$ & 0.080 & - & - \\
\hline APACHE II score & $1.437(1.243-1.636)$ & $<0.001$ & - & - \\
\hline
\end{tabular}

$A B G$ and PBG were reported as per the change in $\mathrm{mmol} / \mathrm{L}$. HbA1c was reported as per change in $1 \%$. SHR, stress hyperglycemia ratio was reported as per change in 0.1. APACHE II score is reported as per 1 change. OR, odds ratio; ABG, admission blood glucose; PBG, postoperative blood glucose.

of the APACHE II score + SHR was significantly greater than that for the APACHE II score alone [0.847 (95\% CI, $0.784-0.911)$ vs. 0.824 (95\% CI, 0.756-0.891); $\mathrm{P}=0.017]$. However, there was no significant difference between the
AUROC curve of 30-day mortality for the APACHE II score + SHR and that for the APACHE II score alone [0.864 (95\% CI, 0.793-0.934) vs. 0.861 (95\% CI, 0.788-0.933); $\mathrm{P}=0.175]$. 
Table 4 Univariable and multivariable regression analysis results showing the relationship between the selected variables and 90 -day mortality

\begin{tabular}{|c|c|c|c|c|}
\hline Risk factor & \multicolumn{2}{|c|}{ Univariable analysis } & \multicolumn{2}{|c|}{ Multivariable analysis } \\
\hline $\mathrm{ABG}, \mathrm{mmol} / \mathrm{L}$ & $0.893(0.567-1.399)$ & 0.108 & - & - \\
\hline PBG, mmol/L & $1.061(1.052-1.480)$ & $<0.001$ & 1.009 (0.928-1.059) & 0.210 \\
\hline $\mathrm{HbA} 1 \mathrm{c}$, per $1 \%$ & $1.653(1.243-2.200)$ & 0.091 & - & - \\
\hline APACHE II score & $1.567(1.028-1.786)$ & 0.001 & - & - \\
\hline
\end{tabular}

ABG (admission blood glucose) and PBG (postoperative blood glucose), postoperative blood glucose were reported as per the change in $\mathrm{mmol} / \mathrm{L}$. HbA1c was reported as per change in $1 \%$. SHR (stress hyperglycemia ratio) ratio was reported as per change in 0.1 . APACHE II score is reported as per 1 change. OR, odds ratio.
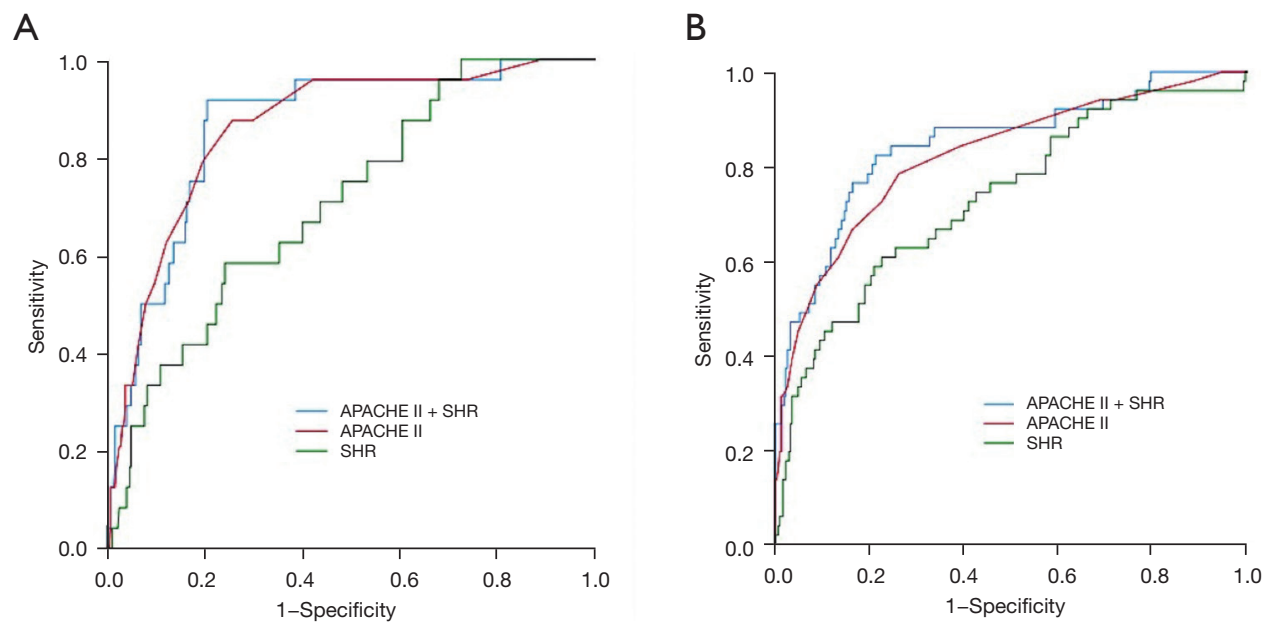

Figure 2 The effect of including the SHR into the 30- and 90-day mortality predictors in ICU. The ROC curves for 30-day mortality (A) and 90-day mortality (B) for SHR, Acute Physiology and Chronic Health Evaluation II (APACHE II), and combined SHR + APACHE II. SHR, stress hyperglycemia ratio; ICU, intensive care unit; ROC, receiver operating characteristic.

\section{Discussion}

This study analyzed data from 365 consecutive critically ill patients who underwent esophagectomy for ESCC. The results showed that SHR $\geq 1.14$ was independently associated with 30-/90-day mortality. By including the APACHE II score, postoperative glucose, and SHR into the multivariable analysis, the relationship between mortality and relative hyperglycemia was still significant, whereas absolute glucose was not. Given that SHR controls background blood glucose, it is a more significant marker of severe postoperative illness than absolute hyperglycemia in the blood glucose spectrum. We also found that SHR performs similarly in predicting short-term mortality in patients with or without diabetes history. In addition, the predictive power of SHR $\geq 1.14$ for anastomotic leakage and cardiovascular diseases was significant in the diabetic population.

According to previous studies, severe stress hyperglycemia may have adverse outcomes in critically ill patients due to direct cellular toxicity $(30,31)$. During acute diseases, stress response including the hypothalamic-pituitary-adrenal axis and the sympathicoadrenal system aim to restore homeostasis. As a result of the consequential interaction of cytokines, catecholamines, and cortisol, an excessive hepatic glucose production, insulin resistance, and glucose tolerance might develop, leading to acute stress hyperglycemia. 
In the central and peripheral neurons, epithelial, hepatocytes, endothelial, and immunological cells, noninsulin-dependent glucose uptake was proportional to blood glucose concentration occurs. It has been supposed that these cells are susceptible to cellular glucose excess, oxidative stress, and consequent damage in the presence of insulin resistance and hyperglycemia $(32,33)$. Furthermore, severe hyperglycemia increases mitochondrial generation of reactive oxygen species while simultaneously compromising the scavenging systems, resulting in ultrastructural and functional anomalies. Previous study has revealed that postoperative hyperglycemia on day three is a predictor of infections following esophagectomy (6). However, another study reported that postoperative hyperglycemia is a risk marker, rather than a risk factor, of increased incidence of infections and prolonged hospital stay in patients undergoing esophagectomy (19). Their study focused on absolute blood glucose values rather than relative blood glucose elevations corrected by SHR. Our study demonstrated a correlation between postoperative glucose levels and 30-/90-day mortality in univariate analysis, but not in multivariate analysis. Therefore, we concluded that in this circumstance, absolute blood glucose cannot be utilized to independently predict mortality.

Prior studies have shown that background blood glucose levels and postoperative hyperglycemia are prognostic risk factors in multiple procedures $(7,12,34,35)$ Okamura $e t$ al. [2017] evaluated the relationship between preoperative HbA1c levels and anastomotic leakage after esophagectomy (35). They identified a significant association between preoperative HbA1c levels and the development of anastomotic leakage following cervical esophagogastric anastomosis. This was the first study to indicate the usefulness of determining the HbA1c levels prior to esophageal surgery. In the second year, Okamura's team evaluated the survival of patients with ESCC following esophagectomy according to their diabetes and glycemic status, as evaluated by using HbA1c levels (36). They found that in individuals with advanced-stage ESCC, poor glycemic control was an independent risk factor for overall and disease-specific mortality following esophagectomy. The discovery of HbAlc is a milestone advance in the history of diabetes research, considering that it can be used as a diagnostic test and can also guide glucose-lowering therapy (37). In this study, we utilized HbA1c to determine background blood glucose, which enabled us to calculate the relative hyperglycemia and quantify it using SHR.

The role of stress hyperglycemia has not been clarified previously, especially in the presence of diabetes. Elevated blood glucose levels during stressful conditions may indicate the presence of stress hyperglycemia in individuals without a history of diabetes. However, the probability of newly diagnosed or unknown diabetes cannot be ruled out. As a result, Roberts et al. (20) introduced the SHR for controlling background blood glucose and recognizing individuals at risk of critical illness with relative hyperglycemia. This is analogous to BMI superiority over body weight as a predictor of health outcomes. The PBG of all patients was easily obtained; therefore, it is convenient to use PBG to calculate the SHR, which facilitates more precise detection and quantification of stress hyperglycemia, and is conducive to risk stratification soon after admission.

The SHR has been used in several studies. In 2017, Yang et al. determined the value of SHR in predicting mortality risk among patients with coronary artery disease (CAD) who had undergone percutaneous coronary intervention (PCI). They observed an elevated mortality risk in the upper SHR quartile of subjects (38). In 2020, a study involving 1,262 consecutive critically ill patients revealed that after correcting for covariables, independently, relative hyperglycemia was related to an increased risk of inhospital mortality. However, mortality was not affected by background glycaemia (30).

In our study, after adjustment for APACHE II score and other glucose-related measures, each 0.1 rise in SHR was found to be associated with a $3-4 \%$ elevation in the OR for short-term mortality. On the face of it, this increment appears insignificant, and its clinical significance may be negligible. However, for a patient with a HbA1c of $5 \%$, a 0.1 -point rise in the SHR represents a $3-4 \%$ increase in the OR of mortality, and the absolute blood glucose of this patient would be $0.55 \mathrm{mmol} / \mathrm{L}(9.9 \mathrm{mg} / \mathrm{dL})$ higher than the basic level calculated according to the SHR formula. From the perspective of absolute blood glucose, an increase of absolute blood glucose by $0.55 \mathrm{mmol} / \mathrm{L}$ is still easily ignored. However, from the perspective of relative blood glucose, the following assumptions can be made. In this study, the range of SHR was $0.80-4.24$, the OR of mortality for the patient with the largest SHR value was $103-137 \%$ higher than that of the patient with the smallest SHR value. Considering that the APACHE II scoring system lacks blood glucose evaluation in critically ill patients, we also investigated whether the addition of SHR may increase the AUROC curve of the APACHE II score. The results indicated that adding the SHR to APACHE II increased the AUROC curve for 90-day mortality prediction by a small 
but significant amount.

Presently, there are still some controversies in postoperative glucose management, such as the target blood glucose range of elective surgery. However, data on the use of an optimized postoperative glucose management target and outcomes in general surgery are still lacking. The American College of Physicians (ACP) suggested a $140-200 \mathrm{mg} / \mathrm{dL}(7.7-11.1 \mathrm{mmol} / \mathrm{L})$ target blood glucose range in surgical and medical ICUs for patients with or without diabetes (39). However, in a retrospective investigation of critically ill patients, a higher glucose target of $5-7.8 \mathrm{mmol} / \mathrm{L}(90-140 \mathrm{mg} / \mathrm{dL})$ had a lower rate of mortality in patients without diabetes, compared with a glucose target of $4.4-6.1 \mathrm{mmol} / \mathrm{L}(80-110 \mathrm{mg} / \mathrm{dL})(40)$. In the non-diabetic population, a moderate blood glucose management strategy will increase surgical site infections, length of hospitalization, as well as the risk of poor wound healing. In contrast, the opposite was observed in diabetic patients; these patients could benefit from moderate blood glucose control and very tight glycemic control could result in a higher incidence of hypoglycemia. This means that postoperative blood glucose management needs to be personalized, and the SHR is an appropriate indicator. It is assumed that the Hba1c of a non-diabetic patient is $5 \%$, excluding the possibility of pre-diabetes (HbA1c of 5.1-6.4\%). According to the formula, the estimated average blood glucose level is $5.4 \mathrm{mmol} / \mathrm{L}$ over the preceding 3 months. In this study, we found that a SHR $<1.14$ could benefit patients, and thus, their blood sugar should be controlled at $4.4-6.2 \mathrm{mmol} / \mathrm{L}$. We also found that for diabetic patients with a HbA1c level of $7 \%$ (with an estimated average blood glucose level of $8.6 \mathrm{mmol} / \mathrm{L}$ ), the suitable blood glucose management goal was $7.7-9.8 \mathrm{mmol} / \mathrm{L}$. Based on this, blood glucose management incorporating the SHR is more personalized and detailed.

This study had some limitations that should be considered. Firstly, our research was an observational study. In this study, we believed that the SHR is a suitable index (as opposed to absolute hyperglycemia) to evaluate the prognosis and individualize treatment in critically ill patients after esophagectomy, but we did not verify this view in a prospective randomized study. Secondly, this was a retrospective study conducted at a single center, and therefore, has a potential selection bias. Furthermore, the statistical power was limited by the small sample size. The study's strength was its precise patient selection, which ensured that the relative elevation in glucose was most likely caused by stress hyperglycemia. To our knowledge, this is the first study to determine that stress hyperglycemia based on the SHR is useful in controlling for background blood glucose in evaluating the prognosis of patients undergoing esophagectomy.

\section{Conclusions}

In summary, a relative increase in blood glucose, as quantified by the SHR, was found to be related to 30-/90-day all-cause mortality in patients who were admitted to the ICU with severe complications after esophagectomy, whereas absolute hyperglycemia was not. A SHR $\geq 1.14$ could be used to identify people at an increased risk of adverse outcomes, particularly diabetic patients, and it may be conveniently calculated using the postoperative glucose and HbA1c levels, and applied to developing and individualizing a glycemic management strategy for all patients.

\section{Acknowledgments}

Funding: None.

\section{Footnote}

Reporting Checklist: The authors have completed the STARD reporting checklist. Available at https://jgo.amegroups.com/ article/view/10.21037/jgo-22-11/rc

Data Sharing Statement: Available at https://jgo.amegroups. com/article/view/10.21037/jgo-22-11/dss

Conflicts of Interest: All authors have completed the ICMJE uniform disclosure form (available at https://jgo.amegroups. com/article/view/10.21037/jgo-22-11/coif). The authors have no conflicts of interest to declare.

Ethical Statement: The authors are accountable for all aspects of the work in ensuring that questions related to the accuracy or integrity of any part of the work are appropriately investigated and resolved. The study was conducted in accordance with the Declaration of Helsinki (as revised in 2013). This study was approved by the Ethics Committees of the Fudan University Shanghai Cancer Center, Shanghai, China. Because this study used retrospective data routinely collected during health screening process, the ethics committee exempted the requirement of obtaining informed consent. 
Open Access Statement: This is an Open Access article distributed in accordance with the Creative Commons Attribution-NonCommercial-NoDerivs 4.0 International License (CC BY-NC-ND 4.0), which permits the noncommercial replication and distribution of the article with the strict proviso that no changes or edits are made and the original work is properly cited (including links to both the formal publication through the relevant DOI and the license). See: https://creativecommons.org/licenses/by-nc-nd/4.0/.

\section{References}

1. Ferlay J, Shin HR, Bray F, et al. Estimates of worldwide burden of cancer in 2008: GLOBOCAN 2008. Int J Cancer 2010;127:2893-917.

2. Devesa SS, Blot WJ, Fraumeni JF Jr. Changing patterns in the incidence of esophageal and gastric carcinoma in the United States. Cancer 1998;83:2049-53.

3. Parkin DM, Bray FI, Devesa SS. Cancer burden in the year 2000: the global picture. Eur J Cancer 2001;37:Suppl 8:S4-S66.

4. Tapias LF, Muniappan A, Wright CD, et al. Short and long-term outcomes after esophagectomy for cancer in elderly patients. Ann Thorac Surg 2013;95:1741-8.

5. Markar SR, Low DE. Physiology, not chronology, dictates outcomes after esophagectomy for esophageal cancer: outcomes in patients 80 years and older. Ann Surg Oncol 2013;20:1020-6.

6. Ito N, Iwaya T, Ikeda K, et al. Hyperglycemia 3 days after esophageal cancer surgery is associated with an increased risk of postoperative infection. J Gastrointest Surg 2014;18:1547-56.

7. Russo N. Perioperative glycemic control. Anesthesiol Clin 2012;30:445-66.

8. Umpierrez GE, Isaacs SD, Bazargan N, et al. Hyperglycemia: an independent marker of in-hospital mortality in patients with undiagnosed diabetes. J Clin Endocrinol Metab 2002;87:978-82.

9. Frisch A, Chandra P, Smiley D, et al. Prevalence and clinical outcome of hyperglycemia in the perioperative period in noncardiac surgery. Diabetes Care 2010;33:1783-8.

10. Kotagal M, Symons RG, Hirsch IB, et al. Perioperative hyperglycemia and risk of adverse events among patients with and without diabetes. Ann Surg 2015;261:97-103.

11. Kwon S, Thompson R, Dellinger P, et al. Importance of perioperative glycemic control in general surgery: a report from the Surgical Care and Outcomes Assessment
Program. Ann Surg 2013;257:8-14.

12. Martin ET, Kaye KS, Knott C, et al. Diabetes and Risk of Surgical Site Infection: A Systematic Review and Metaanalysis. Infect Control Hosp Epidemiol 2016;37:88-99.

13. Capes SE, Hunt D, Malmberg K, et al. Stress hyperglycaemia and increased risk of death after myocardial infarction in patients with and without diabetes: a systematic overview. Lancet 2000;355:773-8.

14. Capes SE, Hunt D, Malmberg K, et al. Stress hyperglycemia and prognosis of stroke in nondiabetic and diabetic patients: a systematic overview. Stroke 2001;32:2426-32.

15. Krinsley JS. Association between hyperglycemia and increased hospital mortality in a heterogeneous population of critically ill patients. Mayo Clin Proc 2003;78:1471-8.

16. Baker EH, Janaway CH, Philips BJ, et al. Hyperglycaemia is associated with poor outcomes in patients admitted to hospital with acute exacerbations of chronic obstructive pulmonary disease. Thorax 2006;61:284-9.

17. Buehler L, Fayfman M, Alexopoulos AS, et al. The impact of hyperglycemia and obesity on hospitalization costs and clinical outcome in general surgery patients. J Diabetes Complications 2015;29:1177-82.

18. Falciglia M, Freyberg RW, Almenoff PL, et al. Hyperglycemia-related mortality in critically ill patients varies with admission diagnosis. Crit Care Med 2009;37:3001-9.

19. Vriesendorp TM, DeVries JH, Hulscher JB, et al. Early postoperative hyperglycaemia is not a risk factor for infectious complications and prolonged inhospital stay in patients undergoing oesophagectomy: a retrospective analysis of a prospective trial. Crit Care 2004;8:R437-R442.

20. Roberts GW, Quinn SJ, Valentine N, et al. Relative Hyperglycemia, a Marker of Critical Illness: Introducing the Stress Hyperglycemia Ratio. J Clin Endocrinol Metab 2015;100:4490-7.

21. Liao WI, Wang JC, Chang WC, et al. Usefulness of Glycemic Gap to Predict ICU Mortality in Critically Ill Patients With Diabetes. Medicine (Baltimore) 2015;94:e1525.

22. Liao WI, Sheu WH, Chang WC, et al. An elevated gap between admission and A1C-derived average glucose levels is associated with adverse outcomes in diabetic patients with pyogenic liver abscess. PLoS One 2013;8:e64476.

23. Su YW, Hsu CY, Guo YW, et al. Usefulness of the plasma glucose concentration-to-HbA1c ratio in predicting clinical outcomes during acute illness with extreme 
hyperglycaemia. Diabetes Metab 2017;43:40-7.

24. Charlson ME, Pompei P, Ales KL, et al. A new method of classifying prognostic comorbidity in longitudinal studies: development and validation. J Chronic Dis 1987;40:373-83.

25. Knaus WA, Draper EA, Wagner DP, et al. APACHE II: a severity of disease classification system. Crit Care Med 1985;13:818-29.

26. Gockel I, Niebisch S, Ahlbrand CJ, et al. Risk and Complication Management in Esophageal Cancer Surgery: A Review of the Literature. Thorac Cardiovasc Surg 2016;64:596-605.

27. Derogar M, Orsini N, Sadr-Azodi O, et al. Influence of major postoperative complications on health-related quality of life among long-term survivors of esophageal cancer surgery. J Clin Oncol 2012;30:1615-9.

28. Nathan DM, Kuenen J, Borg R, et al. Translating the A1C assay into estimated average glucose values. Diabetes Care 2008;31:1473-8.

29. Di Luzio R, Dusi R, Mazzotti A, et al. Stress Hyperglycemia and Complications Following Traumatic Injuries in Individuals With/Without Diabetes: The Case of Orthopedic Surgery. Diabetes Metab Syndr Obes 2020;13:9-17.

30. Lee TF, Drake SM, Roberts GW, et al. Relative Hyperglycemia Is an Independent Determinant of InHospital Mortality in Patients With Critical Illness. Crit Care Med 2020;48:e115-22.

31. Fabbri A, Marchesini G, Benazzi B, et al. Stress Hyperglycemia and Mortality in Subjects With Diabetes and Sepsis. Crit Care Explor 2020;2:e0152.

32. Van den Berghe G. How does blood glucose control with insulin save lives in intensive care? J Clin Invest 2004;114:1187-95.

Cite this article as: Xia Z, Gu T, Zhao Z, Xing Q, Zhang Y, Zhang Z, Zhu B. The stress hyperglycemia ratio, a novel index of relative hyperglycemia, predicts short-term mortality in critically ill patients after esophagectomy. J Gastrointest Oncol 2022;13(1):56-66. doi: 10.21037/jgo-22-11
33. Brownlee M. Biochemistry and molecular cell biology of diabetic complications. Nature 2001;414:813-20.

34. Lee W, Yoon YS, Han HS, et al. Prognostic relevance of preoperative diabetes mellitus and the degree of hyperglycemia on the outcomes of resected pancreatic ductal adenocarcinoma. J Surg Oncol 2016;113:203-8.

35. Okamura A, Watanabe M, Imamura Y, et al. Preoperative Glycosylated Hemoglobin Levels Predict Anastomotic Leak After Esophagectomy with Cervical Esophagogastric Anastomosis. World J Surg 2017;41:200-7.

36. Okamura A, Watanabe M, Imamura Y, et al. Glycemic Status and Prognosis of Patients with Squamous Cell Carcinoma of the Esophagus. World J Surg 2017;41:2591-7.

37. Chamberlain JJ, Rhinehart AS, Shaefer CF Jr, et al. Diagnosis and Management of Diabetes: Synopsis of the 2016 American Diabetes Association Standards of Medical Care in Diabetes. Ann Intern Med 2016;164:542-52.

38. Yang Y, Kim TH, Yoon KH, et al. The stress hyperglycemia ratio, an index of relative hyperglycemia, as a predictor of clinical outcomes after percutaneous coronary intervention. Int J Cardiol 2017;241:57-63.

39. Qaseem A, Humphrey LL, Chou R, et al. Use of intensive insulin therapy for the management of glycemic control in hospitalized patients: a clinical practice guideline from the American College of Physicians. Ann Intern Med 2011;154:260-7.

40. Lanspa MJ, Hirshberg EL, Phillips GD, et al. Moderate glucose control is associated with increased mortality compared with tight glucose control in critically ill patients without diabetes. Chest 2013;143:1226-34.

(English Language Editor: A. Kassem) 